\title{
A Handshake-based Protocol Exploiting the Near-Far Effect in Underwater Acoustic Networks
}

\author{
Roee Diamant, Paolo Casari, Senior Member, IEEE, Filippo Campagnaro, Michele Zorzi, Fellow, IEEE
}

\begin{abstract}
We propose a scheme that opportunistically exploits received power diversity across different packets to both favor spatial reuse in underwater acoustic networks and limit the exposed terminal problem. Since the power attenuation in the underwater acoustic channel is large, such power differences are common. This situation, also referred to as the near-far effect and often considered a problem, is converted into a resource by means of multipacket reception (MPR). Yet, even without MPR capability our scheme offers performance benefits. Our scheme is simple, lightweight and distributed, and can be easily implemented over any protocol based on carrier-sense multiple access with collision avoidance. Our results show that higher throughput and lower transmission delay are achieved compared to a benchmark channel access protocol. Our results are validated and demonstrated in a lake experiment. To allow reproducibility, the implementation of our scheme is publicly available.

Index Terms-Underwater acoustic networks; near-far effect; spatial reuse; handshaking, collision avoidance; long propagation delay; simulations; lake experiment
\end{abstract}

\section{INTRODUCTION}

$\mathbf{T}$ HE design of medium access control (MAC) protocols for contention-based communications in underwater acoustic networks (UWANs) is challenged by long propagation delays and low physical transmission rates, which represent significant bottlenecks for resource assignment even in small networks. In addition, the absorption loss in the underwater channel leads to large differences in acoustic power attenuation for relatively small changes in the transmission range. As a result, with respect to a common receiver, the transmissions of a closer node may be much stronger, and thus be received while jamming those of a farther node. For contentionbased communications based on the Multiple Access Collision Avoidance (MACA) protocol, this near-far effect (NFE) compounds as Request-To-Send (RTS)/Clear-To-Send (CTS) packets are exposed to collisions. The NFE is typically compensated in MAC protocol design via power control [1] or mechanisms that avoid simultaneous transmissions by nodes belonging to the same near-far node set (NFNS) [2]. However, the former requires a centralized control, which due to the long propagation delay should be avoided for contention-based UWANs. On the other hand, since the NFE is so common in UWANs, avoiding simultaneous transmissions within the same NFNS greatly reduces the network throughput.

In this paper, we take a different approach, and actually exploit information on NFNSs to increase the network perfor-

Manuscript received: February 8, 2016; accepted: March 24, 2016.

The associate editor coordinating the review of this letter and approving it for publication was Hai Jiang.

Roee Diamant (corresponding author, email: roeed@univ.haifa.ac.il), Filippo Campagnaro, and Michele Zorzi are with the Department of Information Engineering, University of Padova, Italy. Paolo Casari is with the IMDEA Networks Institute, Madrid, Spain.

This work has been supported in part by the US Office of Naval Research under Grant no. N62909-14-1-N127.

Digital Object Identifier: XX.XXXX/WCL... mance of MACA-based protocols. Specifically, we argue that packets from a NFNS can be received simultaneously, e.g., by means of multipacket reception (MPR) via interference cancellation, which has been advantageously applied to underwater communications [3], [4]. Even without MPR, simultaneous transmissions by nodes in the same NFNS can still be permitted if their destinations are different. Considering these opportunities, we offer a near-far handshake (NF-Handshake) scheme that exploits the NFE in UWANs (for the first time, to the best of our knowledge). Our scheme is simple, lightweight, distributed, and can be easily implemented over any MACAbased protocol. Our simulations, validated by results from a lake experiment, demonstrate that, at the expense of a small fairness reduction, NF-Handshake achieves better throughput and delivery delay with respect to a benchmark scheme.

\section{RELATED WORK}

Most contention-based channel access schemes for UWANs are inspired by the MACA approach. MACA prescribes that data transmissions be preceded by an RTS/CTS handshake, meant to protect the upcoming transmission from interference. Slotted-FAMA [5] improves this concept by mandating that transmissions take place at the beginning of global time slots. Conversely, no time slotting is employed in DACAP [6], which is based on collision detection mechanisms.

Several extensions of MACA have been proposed. Assuming global propagation delay information, MACA-MN [7] employs simultaneous transmissions of RTS packets to multiple receivers, and schedules multiple data packets for different receivers back-to-back. BiC-MAC [8] exploits bi-directional point-to-point data transmission chances. RIPT [9] schedules data packet transmissions from multiple nodes to arrive at the same receiver back-to-back, assuming at least local propagation delay knowledge. DOTS [2] assumes networkwide synchronization and allows the nodes to engage in multiple handshakes by distributedly collecting propagation delay information. MAC and error control are jointly designed in SASHA [10]. In TSR [11], potentially interfering communications can co-exist by timing transmissions to exploit silence periods, and by adaptively tuning the size of the data packets.

In this paper, we propose a fully distributed MAC scheme that can augment any MACA-based protocol by taking advantage of the NFE in UWANs with and without MPR capabilities. Our scheme keeps the communication overhead to a minimum and does not require any knowledge of propagation delays or two-hop connectivity.

\section{The NF-HandshaKe Scheme}

We assume a UWAN where any node can start a communication session with one of its one-hop neighbors. Each node is assumed to hold up-to-date information about its onehop neighbor list and its NFNSs. In NF-handshake, CTSs are 
directed not just to the source of a received RTS, but rather to all nodes which can potentially transmit together with that same source. More specifically, for a source node $n$ from which an RTS packet was received, the receiver $r$ checks for NFNSs including node $n$. Among these NFNSs, receiver $r$ identifies the best subset such that channel utilization is maximized, and piggybacks this information in its broadcast CTS packet, e.g., by means of a bit map. Upon receiving a CTS, any node $j$ whose identity was included in the CTS has an option to transmit instead of remaining silent (which otherwise is enforced upon overhearing the RTS of node $n$ or the CTS of node $r$ ). The direct result is a mitigation of the exposed terminal problem. In the following we will explain how NFNSs are determined, and provide the rules of NFHandshake with and without MPR.

\section{A. Determining NFNSs}

The information about NFNPs is obtained distributedly by each node. This information can be inferred from incoming message statistics [12] or, given the nodes' distances, it can be computed via an attenuation model [2]. In this work, we determine the NFNSs relative to each node by continuously measuring the power of each received signals and by setting a threshold on the calculated signal-to-interference-plus-noise ratio (SINR). To formalize this, let $\boldsymbol{g}_{n}(r)$ be a possible NFNS with respect to receiver $r$ including node $n$, from which the first RTS message was received. Also, call $P_{r, j}$ the received power of the latest signal received from node $j$ at node $r$, and $\eta_{r}$ the noise power observed by node $r$. For set $\boldsymbol{g}_{n}(r)$, the expected SINR of packets from node $n$ is

$$
\mu_{r, n}=P_{r, n}\left[\eta_{r}+\sum_{i \in \boldsymbol{g}_{n}(r), i \neq n} P_{r, i}\right]^{-1} .
$$

Note that (1) is a worst-case assumption, since it is unlikely that all nodes in $\boldsymbol{g}_{n}(r)$ will transmit simultaneously. However, without propagation delay information, it cannot be avoided. By recalculating $\mu_{r, n}$, we can compensate for mobility or received power changes. However, such changes should be sufficiently slow, so that the network topology information remains accurate. Still, we remark that NF-Handshake is distributed, hence even upon fast changes in $\mu_{r, n}$, only the communication to and from the node whose topology information is outdated would be affected.

Given the SINR $\mu_{r, n}$, the modulation scheme, the packet length, and the processing and coding gains, we calculate the expected error probability $p\left(\boldsymbol{g}_{n}(r)\right)$ for a packet from node $n$ received together with packets from nodes in $\boldsymbol{g}_{n}(r)$ [12]. In case $p\left(\boldsymbol{g}_{n}^{n}(r)\right)$ is greater than a target packet reception rate, NFNS $\boldsymbol{g}_{n}(r)$ is considered valid. Similarly, node $r$ determines the set of all valid NFNSs of maximal cardinality, $\boldsymbol{G}_{n}(r)$. Since (1) is calculated for the latest $P_{r, i}, i \in \boldsymbol{g}_{n}(r)$ measurements, set $\boldsymbol{G}_{n}(r)$ is continuously updated every time an RTS from a node $n$ is received and mobility is permitted. Finding $G_{n}(r)$ in general is NP-hard with complexity $\mathcal{O}\left(2^{D}-1\right)$, where $D$ is the network degree. However, similar to the branch-and-bound algorithm [13], the average complexity is $\mathcal{O}\left(D^{3}\right)$. If $D$ is large, a suboptimal computation of $\boldsymbol{g}_{n}(r) \in$ $\boldsymbol{G}_{n}(r)$ can be performed by subsequently adding nodes in ascending order of received power, with complexity $\mathcal{O}\left(N^{2}\right)$.

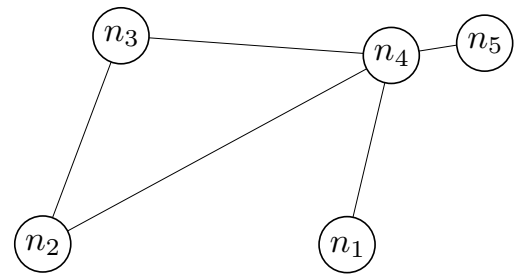

Fig. 1. Reference scenario with $N=5$ nodes for illustrating NF-Handshake.

In its CTS packet, node $r$ grants permission to transmit to the nodes of a selected subset $\boldsymbol{g}_{n}^{\star}(r) \in \boldsymbol{G}_{n}(r)$. For a fair allocation, each valid NFNS $\boldsymbol{g}_{n}^{i}(r) \in \boldsymbol{G}_{n}(r)$ is associated a selection probability as

$$
p_{\mathrm{ch}}\left(\boldsymbol{g}_{n}^{i}(r)\right)=p\left(\boldsymbol{g}_{n}^{i}(r)\right)\left[\sum_{\boldsymbol{g}_{n}^{j}(r) \in \boldsymbol{G}_{n}(r)} p\left(\boldsymbol{g}_{n}^{j}(r)\right)\right]^{-1},
$$

and $\boldsymbol{g}_{n}^{\star}(r)$ is chosen at random according to such probabilities.

\section{B. NF-Handshake Rules}

Assume node $n$ sent an RTS, and node $r$ answered with a CTS. If this CTS includes node $i$ 's ID, node $i$ does not enter a silent state. Instead, if its queue is not empty, node $i$ can exploit the opportunity to transmit. The rules to do so change depending on the availability of MPR capabilities.

Without MPR capabilities - In this case, $i$ cannot transmit a data packet to node $r$ or node $n$. Still, in case node $i$ was not silenced by a communication session other than that between $r$ and $n, i$ is free to transmit an RTS message to another destination node, or to send a CTS in response to an RTS.

With MPR capabilities - In this case, node $i$ can either transmit an RTS or a CTS to a node different than $r$ and $n$, or directly transmit a data message to node $r$. Since $r$ already considered the interference from node $i$, the latter is expected not to interfere with the transmission of node $n$, and node $r$ will try to decode both transmissions via its MPR capabilities.

\section{NF-Handshake's behavior with MPR}

Refer to the sample network topology in Fig. 1, with $N=5$ nodes named $n_{1}$ to $n_{5}$. Besides the trivial case in which an RTS/CTS/data exchange is completed without further interactions, consider the following four scenarios:

Case 1 (compatible concurrent transmitters): assume that node $n_{3}$ completes an RTS/CTS handshake to transmit a packet to $n_{2}$. If $n_{4}$ also has a packet for $n_{2}$, it will check if its ID is included in $n_{2}$ 's CTS. If this is the case, it means that $n_{4} \in \boldsymbol{g}_{n_{3}}^{\star}\left(n_{2}\right)$, and $n_{4}$ can send its packet to $n_{2}$ as well.

Case 2 (mutually incompatible concurrent transmitters): assume that, e.g., $n_{3}$ and $n_{5}$ have a packet for $n_{4}$ and transmit an RTS to it. Assume that $n_{4}$ can successfully receive the two RTSs, but $n_{3} \notin \boldsymbol{g}_{n_{5}}^{\star}\left(n_{4}\right)$ and $n_{5} \notin \boldsymbol{g}_{n_{3}}^{\star}\left(n_{4}\right)$. In this case, $n_{4}$ grants $n_{3}$ in the CTS with probability $p_{3} /\left(p_{3}+p_{5}\right)$ and conversely grants $n_{5}$ with probability $p_{5} /\left(p_{3}+p_{5}\right)$, where $p_{3}=p_{\mathrm{ch}}\left(\boldsymbol{g}_{n_{3}}^{\star}\left(n_{4}\right)\right)$ and $p_{5}=p_{\mathrm{ch}}\left(\boldsymbol{g}_{n_{5}}^{\star}\left(n_{4}\right)\right)$.

Case 3 (partially incompatible concurrent transmitters): assume that $n_{3}$ sends an RTS to $n_{4}$ and that $n_{5}$ and $n_{1}$ also send an RTS. Assume that $\left\{n_{1}, n_{5}\right\} \not \subset \boldsymbol{g}_{n_{3}}^{\star}\left(n_{4}\right)$. Then, node $n_{4}$ would grant transmission to $n_{3}$ and either $n_{1}$ or $n_{5}$. Node $n_{1}$ is selected with probability $p_{1} /\left(p_{1}+p_{5}\right)$ and node $n_{5}$ with probability $p_{5} /\left(p_{1}+p_{5}\right)$, where $p_{1}=p_{\text {ch }}\left(\boldsymbol{g}_{n_{3}}^{x}\left(n_{4}\right)\right)$, 


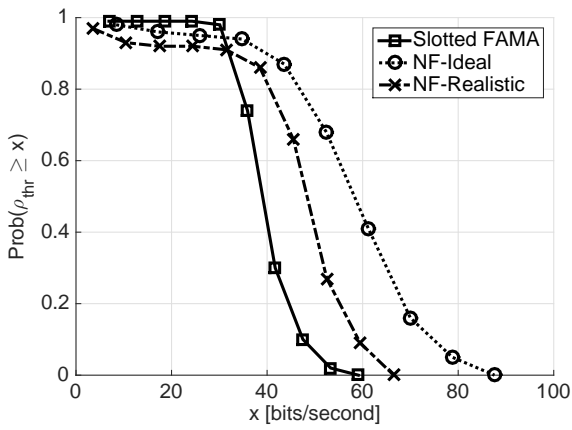

Fig. 2. Empirical C-CDF of $\rho_{\mathrm{thr}}$ from (3). $N=10, T=500 \mathrm{~s}, N_{\text {bit }}=1000$ bits.

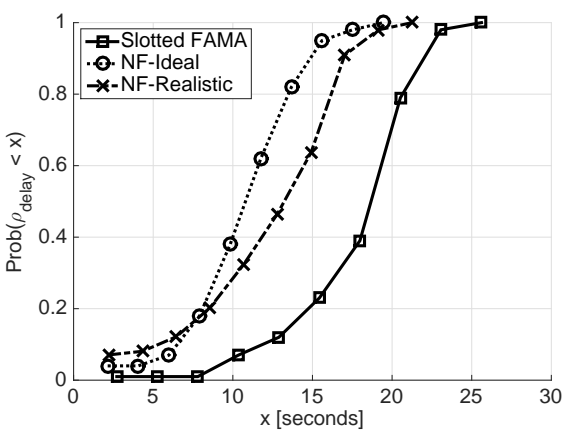

Fig. 3. Empirical $\mathrm{CDF}$ of $\rho_{\text {delay }}$ from (4). $N=10, T=500 \mathrm{~s}, N_{\text {bit }}=1000$ bits

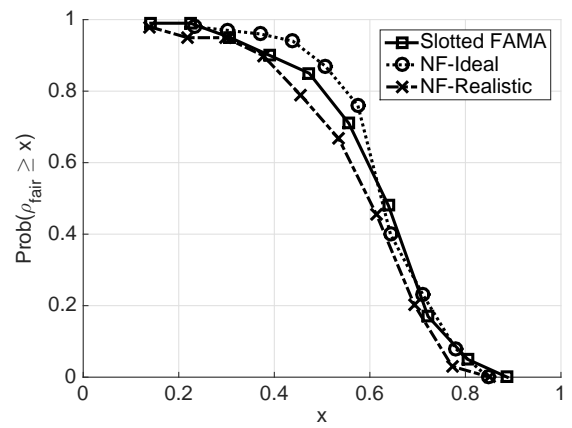

4). Fig. 4. Empirical C-CDF of $\rho_{\text {fair from (5). }}$ $N=10, T=500 \mathrm{~s}, N_{\text {bit }}=1000$ bits. $n_{1} \in \boldsymbol{g}_{n_{3}}^{x}\left(n_{4}\right)$, and $p_{5}=p_{\text {ch }}\left(\boldsymbol{g}_{n_{3}}^{y}\left(n_{4}\right)\right), n_{5} \in \boldsymbol{g}_{n_{3}}^{y}\left(n_{4}\right)$, for some selected subset indices $x$ and $y$.

Case 4 (two handshakes with different destinations): assume that node $n_{2}$ sends an RTS to $n_{3}$ and node $n_{1}$ sends an RTS to $n_{4}$. Typically, $n_{3}$ and $n_{4}$ will answer independently with their own CTS. Overhearing both CTS packets, $n_{2}$ will continue to send its data packet to $n_{3}$ if it is included in the CTS of $n_{4}$, and $n_{1}$ will do the same if it appears in the CTS of $n_{3}$. If both nodes are blocked by the CTSs, only the communication session whose CTS arrived first will continue.

\section{Simulation Results}

Our simulations involve a Monte-Carlo set of 200 runs. In each run, we generate a topology of $N=10$ nodes, deployed uniformly at random over an area of $5 \times 5 \mathrm{~km}^{2}$, with depth of $100 \mathrm{~m}$. Four horizontal and one vertical obstacles of length uniformly distributed in $[100,200] \mathrm{m}$ are placed at random locations. For each possible link, we compute the transmission loss via the Bellhop ray tracing model [14]. Assuming the use of BPSK, we compute the expected bit error rate for a bit rate of $1000 \mathrm{bps}$, a source level of $150 \mathrm{~dB}$ re $1 \mu \mathrm{Pa} @ 1 \mathrm{~m}$ and a noise level of $50 \mathrm{~dB}$ re $(1 \mu \mathrm{Pa} / \mathrm{Hz})$. A link is considered to be feasible if this probability is less than $10^{-3}$, and if no obstacle intersects the link. Packets are generated according to a Poisson process of rate 0.01 packets/s/node. Data packets are 1000-bit long, and RTSs and CTSs consist of 20 bits.

Our NF-handshake scheme can be implemented over any MACA-based protocol. To evaluate its performance, we choose to implement it over Slotted-FAMA [5], a widely known approach where the transmission of RTS, CTS and data packets is scheduled at the beginning of global time slots. To avoid collisions, each time slot includes a guard interval that is sufficiently long to compensate both for possible clock drifts and for the maximum propagation delay between any two nodes. For our simulations, this leads to time slots of duration $4.43 \mathrm{~s}$. In UWANs, the propagation delay is significantly large compared to the ambiguity in time synchronization, hence the effect of such guard intervals on network performance is negligible. We publish our implementation ${ }^{1}$ for reproducibility.

We consider two types of communication systems: i) $N F$ Ideal, where for each NFNS both the jammer and the jammed nodes are decoded with probability 1 , and ii) NF-Realistic, where the MPR probability is set according to SINR values. The case where the MPR capability is not available is

\footnotetext{
${ }^{1} \mathrm{http}: / /$ marsci.haifa.ac.il/share/diamant/NFHandshakeCode.zip
}

considered for the lake experiment below. We compare the performance of the two schemes to that of plain Slotted-FAMA in terms of throughput, delivery delay, and service fairness. Defining $x_{n, m}$ as the number of data packets generated by node $n$ for node $m$ and successfully received by the latter, $T$ as the simulation time, and $N_{\text {bit }}$ as the number of information bits in a single data packet, the total network throughput is

$$
\rho_{\mathrm{thr}}=\frac{1}{T} \sum_{n=1}^{N} \sum_{\substack{m=1 \\ m \neq n}}^{N} x_{n, m} N_{\mathrm{bit}} \text {. }
$$

In Fig. 2, we show the empirical complementary cumulative distribution function (C-CDF) of $\rho_{\mathrm{thr}}$ for $T=500 \mathrm{~s}$. The results show that even without perfect MPR capability, our NF-Handshake scheme significantly increases the throughput. We also observe that the dispersion of $\rho_{\mathrm{thr}}$ is about the same for Slotted-FAMA and NF-Handshake. This verifies that the throughput increase is achieved for different network settings.

Let $t_{n, m, j}$ be the delay from the time a data packet $j$ is transferred to the MAC layer of source $n$ till it is successfully delivered to its destination node $m$. Note that $t_{n, m, j}$ captures both the queuing and the end-to-end transmission delay. The average delivery delay (in s) is defined as

$$
\rho_{\text {delay }}=\frac{1}{N(N-1)} \sum_{n=1}^{N} \sum_{\substack{m=1 \\ m \neq n}}^{N} \frac{1}{x_{n, m}} \sum_{j=1}^{x_{n, m}} t_{n, m, j} .
$$

In Fig. 3, we show the CDF of $\rho_{\text {delay }}$. We observe a significant improvement of more than $40 \%$ when MPR is limited and $80 \%$ when MPR is ideal. The improvement in both $\rho_{\text {thr }}$ and $\rho_{\text {delay }}$ shown for our scheme translates into better channel utilization.

Last, we evaluate fairness according to Jain's index as

$$
\rho_{\text {fair }}=\left(\sum_{n=1}^{N} \sum_{\substack{m=1 \\ m \neq n}} x_{n, m}\right)^{2}\left[N \sum_{n=1}^{N}\left(\sum_{\substack{m=1 \\ m \neq n}}^{N} x_{n, m}\right)^{2}\right]^{-1} \text {. }
$$

Empirical C-CDF results for $\rho_{\text {fair }}$ are shown in Fig. 4. Compared to Slotted-FAMA we observe that $\rho_{\text {fair }}$ is not affected for ideal MPR and decreases less than $5 \%$ for realistic MPR. The latter result is because nodes involved in favorable NFE scenarios are allowed to transmit more often.

\section{LAKE EXPERIMENT}

We evaluated NF-Handshake in a sea experiment using off-the-shelf underwater acoustic modems. This also demonstrates that our scheme can be easily implemented on top of proprietary architectures. Our experiment was conducted in Dec. 2015 along the eastern coast of the Garda lake, Italy. We 


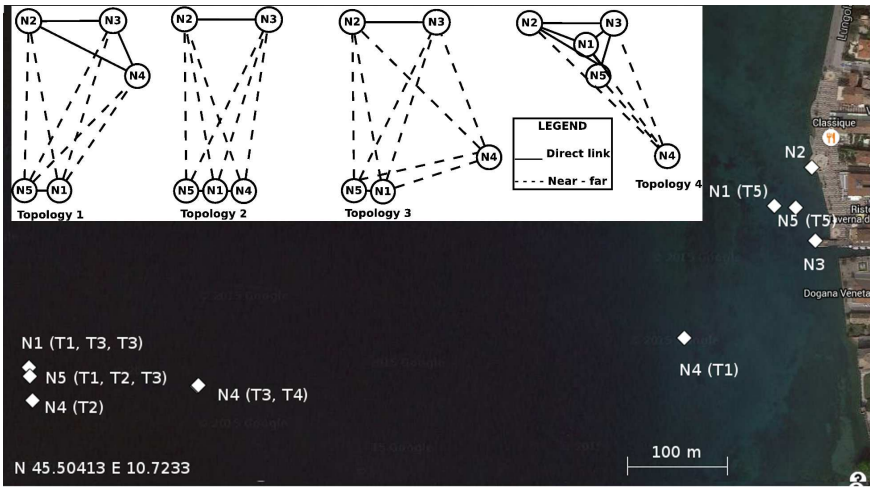

Fig. 5. Lake experiment: dotted lines indicate near-far connections. "N"s are locations of the nodes for each topology ("T").

employed five nodes, communicating via EvoLogics modems, which have no MPR capabilities. The nodes were deployed from two harbor docks and boats. By relocating the boats, we created the network topologies shown in Fig. 5. Nodes 2 and 3 were deployed from harbor docks $75 \mathrm{~m}$ from each other, and at water depth $2 \mathrm{~m}$ and $4 \mathrm{~m}$, respectively. Nodes 1 and 5 were placed $10 \mathrm{~m}$ apart and were deployed at depth $10 \mathrm{~m}$ and at distance $\approx 700 \mathrm{~m}$ from node 2 (Topologies T1, T2, T3) and at water depth $2 \mathrm{~m}$ and distance $\approx 50 \mathrm{~m}$ from node 2 (Topology T4). Node 4 was deployed from an additional boat at water depth $5 \mathrm{~m}$, at $200 \mathrm{~m}$ (Topology T1), $700 \mathrm{~m}$ (Topology $\mathrm{T} 2$ ), and $600 \mathrm{~m}$ (Topologies T3, T4) from node 2. When the boats were far from the harbor, the water depth was 20 to $25 \mathrm{~m}$. Conversely, the depth near the docks was 2 to $5 \mathrm{~m}$. The shallow water and the many reflections from concrete harbor walls resulted in high packet error rates of $\approx 30 \%$.

We employed a slotted handshake framework with time slot duration $10 \mathrm{~s}$. In each topology, we allowed the nodes to communicate for $5 \mathrm{~min}$ using the NF-Handshake scheme, and for $5 \mathrm{~min}$ using the Slotted FAMA protocol. Table I summarizes the results in terms of the ratio of serviced to generated packets and of the number of resolved exposed terminal problems. The latter was measured by counting the number of switches from "silent" to "active" due to a CTS packet granting transmission. We observe that, out of the 78 generated data packets, about $40 \%$ were serviced using the Slotted-FAMA protocol. This is mostly because the full network connectivity resulted in high number of exposed terminal problems and not all generated packets could be serviced. Our NF-Handshake algorithm improves this situation, as it services roughly $68 \%$ of the generated packets. Our algorithm solved on average about four exposed terminal problems per node, i.e., each node received on average the opportunity to participate in four additional communication sessions. It could be argued that such a large number of serviced packets over the same time span may have resulted in much larger interference. However, the comparable average scheduling delay between NF-Handshake (42 s) and Slotted FAMA (37 s) shows that this interference is well managed by our NF-Handshake scheme.

\section{CONCLUSIONS}

We presented a simple distributed scheme to increase the performance of handshake-based UWANs. Our algorithm ex-
TABLE I

RESULTS FROM THE LAKE EXPERIMENT. RATIOS INDICATE SERVICED VS. GENERATED PACKETS. THE PARENTHESES ENCLOSE THE NUMBER OF EXPOSED TERMINAL PROBLEMS RESOLVED.

\begin{tabular}{ccccccc}
\hline Topology & Method & Node 1 & Node 2 & Node 3 & Node 4 & Node 5 \\
\hline \multirow{2}{*}{ T1 } & NF & $3 / 5(5)$ & $3 / 3(6)$ & $1 / 2(3)$ & $1 / 3(1)$ & $1 / 3(4)$ \\
\cline { 2 - 7 } & FAMA & $1 / 5$ & $2 / 3$ & $1 / 2$ & $1 / 3$ & $1 / 3$ \\
\hline \multirow{2}{*}{ T2 } & NF & $2 / 4(5)$ & $2 / 2(6)$ & $5 / 5(5)$ & $5 / 7(5)$ & $2 / 3(5)$ \\
\cline { 2 - 7 } & FAMA & $2 / 4$ & $2 / 2$ & $3 / 5$ & $2 / 7$ & $1 / 3$ \\
\hline \multirow{2}{*}{ T3 } & NF & $1 / 2(4)$ & $1 / 2(3)$ & $4 / 5(5)$ & $4 / 7(5)$ & $4 / 4(3)$ \\
\cline { 2 - 7 } & FAMA & $1 / 2$ & $0 / 2$ & $2 / 5$ & $3 / 7$ & $2 / 4$ \\
\hline \multirow{2}{*}{ T4 } & NF & $3 / 5(3)$ & $2 / 2(4)$ & $4 / 5(3)$ & $2 / 4(4)$ & $3 / 5(4)$ \\
\cline { 2 - 7 } FAMA & $1 / 5$ & $1 / 2$ & $3 / 5$ & $2 / 4$ & $1 / 5$ \\
\hline \multirow{2}{*}{ Total } & \multicolumn{7}{c}{ NF: $53 / 78(83)$} & FAMA: $32 / 78$ \\
\hline
\end{tabular}

ploits information on NFNSs, and allows multiple nodes to transmit simultaneously while avoiding collisions. To keep the communication overhead to a minimum, the identity of the nodes allowed to transmit is piggybacked in CTS packets. This allows communication sessions to co-exist and mitigates the exposed terminal problem. Numerical results show the benefit brought by our scheme in terms of throughput and delivery delay. A lake experiment was performed to validate these results and to demonstrate the effectiveness of our algorithm.

\section{REFERENCES}

[1] D. Pompili, T. Melodia, and I. F. Akyildiz, "A CDMA-based medium access control for underwater acoustic sensor networks," IEEE Trans. Wireless Commun., vol. 8, no. 4, pp. 1899-1909, Apr. 2009.

[2] Y. Noh et al., "DOTS: A propagation delay-aware opportunistic MAC protocol for mobile underwater networks," IEEE Trans. Mobile Comput., vol. 13, no. 4, pp. 766-782, Apr. 2014.

[3] S. E. Cho, H.-C. Song, and W. S. Hodgkiss, "Successive interference cancellation for underwater acoustic communications," IEEE J. Ocean. Eng., vol. 36, no. 4, pp. 490-501, Oct. 2011.

[4] J. McGee, J. Catipovic, S. Schoenecker, and P. Swaszek, "Interference suppression in congested undersea environments," in Proc. MTS/IEEE OCEANS, Genova, Italy, May 2015.

[5] M. Molins and M. Stojanovic, "Slotted FAMA: a MAC Protocol for underwater acoustic networks," in Proc. IEEE Oceans, Singapore, 2006.

[6] B. Peleato and M. Stojanovic, "Distance aware collision avoidance protocol for ad hoc underwater acoustic sensor networks," IEEE Commun. Lett., vol. 11, no. 12, pp. 1025-1027, Dec. 2007.

[7] N. Chirdchoo, W.-S. Soh, and K. C. Chua, "MACA-MN: A MACAbased MAC protocol for underwater acoustic networks with packet train for multiple neighbors," in Proc. IEEE VTC, Singapore, May 2008.

[8] H.-H. Ng, W.-S. Soh, and M. Motani, "A bidirectional-concurrent MAC protocol with packet bursting for underwater acoustic networks," IEEE J. Ocean. Eng., vol. 38, no. 3, pp. 547-565, Jul. 2013.

[9] N. Chirdchoo, W.-S. Soh, and K. Chua, "RIPT: A receiver-initiated reservation-based protocol for underwater acoustic networks," IEEE J. Sel. Areas Commun., vol. 26, no. 9, pp. 1744-1753, Dec. 2008.

[10] H. Mo et al., "Evaluating selective ARQ and slotted handshake based access in real world underwater networks," in Lecture Notes in Computer Science, K. Ren et al., Ed. Springer, 2013, vol. 7992, pp. 206-220.

[11] R. Diamant, W. Shi, W.-S. Soh, and L. Lampe, "Joint time and spatial reuse handshake protocol for underwater acoustic communication networks," IEEE J. Ocean. Eng., vol. 38, no. 3, pp. 470-483, Jul. 2013.

[12] R. Diamant and L. Lampe, "Spatial reuse time-division multiple access for broadcast ad hoc underwater acoustic communication networks," IEEE J. Ocean. Eng., vol. 36, no. 2, pp. 172-185, Feb. 2011.

[13] W. Zhang and R. E. Korf, "An average-case analysis of branch-andbound with applications: Summary of results," AAAI, pp. 545-550, Feb. 1992.

[14] M. Porter et al., "Bellhop code," Last time accessed: Nov. 2015. [Online]. Available: http://oalib.hlsresearch.com/Rays/index.html 